PROCEEDINGS OF THE

AMERICAN MATHEMATICAL SOCIETY

Volume 138, Number 1, January 2010, Pages 355-365

S 0002-9939(09)10028-X

Article electronically published on September 3, 2009

\title{
EXAMPLES OF SMOOTH MAPS WITH FINITELY MANY CRITICAL POINTS \\ IN DIMENSIONS $(4,3),(8,5)$ AND $(16,9)$
}

LOUIS FUNAR, CORNEL PINTEA, AND PING ZHANG

(Communicated by Paul Goerss)

\begin{abstract}
We consider manifolds $M^{2 n}$ which admit smooth maps into a connected sum of $S^{1} \times S^{n}$ with only finitely many critical points, for $n \in$ $\{2,4,8\}$, and compute the minimal number of critical points.
\end{abstract}

\section{Introduction AND STATEMENT OF THE MAIN RESUlt}

Let $\varphi\left(M^{m}, N^{n}\right)$ denote the minimal number of critical points of smooth maps between the manifolds $M^{m}$ and $N^{n}$. When superscripts are specified they denote the dimension of the respective manifolds. We are interested below in the case when $m \geq n \geq 2$ and the manifolds are compact. The main problem concerning $\varphi$ is to characterize those pairs of manifolds for which it is finite non-zero and then to compute its value (see [1]).

In 1 the authors found that, in small codimension $0 \leq m-n-1 \leq 3$, if $\varphi\left(M^{m}, N^{n+1}\right)$ is finite, then $\varphi\left(M^{m}, N^{n+1}\right) \in\{0,1\}$, except for the exceptional pairs of dimensions $(m, n+1) \in\{(2,2),(4,3),(4,2),(5,2),(6,3),(8,5)\}$. Notice that $(5,3)$ was inadvertently included in 1 among the exceptional pairs. Moreover, under the finiteness hypothesis, $\varphi(M, N)=1$ if and only if $M$ is the connected sum of a smooth fibration over $N$ with an exotic sphere and not a fibration itself. There are two essential ingredients in this result. First, there are local obstructions to the existence of isolated singularities; namely, the germs of smooth maps $\mathbb{R}^{m} \rightarrow$ $\mathbb{R}^{n}$ having an isolated singularity at the origin are actually locally topologically equivalent to a projection. Thus, these maps are topological fibrations. Second, singular points located in a disk cluster together.

The simplest exceptional case is that of (pairs of) surfaces, which is completely understood by elementary means (see [2] for explicit computations). Very little is known for the other exceptional and generic (i.e. $m-n-1 \geq 4$ ) cases and even the case of pairs of spheres is still unsettled. In particular, it is not known whether $\varphi$ is bounded in terms only of the dimensions, in general.

The aim of this paper is to find non-trivial examples in dimensions $(4,3),(8,5)$ and $(16,9)$ inspired by the early work of Antonelli $(3,4])$. The smooth maps

Received by the editors July 21, 2008, and, in revised form, April 28, 2009.

2000 Mathematics Subject Classification. Primary 57R45, 55R55, 58K05, 57R60, 57R70.

Key words and phrases. Critical point, isolated singularity, Hopf fibration, suspension, homotopy sphere. 
considered in 44 are so-called Montgomery-Samelson fibrations with finitely many singularities where several fibers are pinched to points. According to [14 these maps should be locally topologically equivalent to a cone over the Hopf fibration, in a neighborhood of a critical point.

The main ingredient of our approach is the existence of global obstructions of a topological nature to the clustering of genuine critical points in these dimensions. This situation seems rather exceptional and it permits us to obtain the precise value of $\varphi$ using only basic algebraic topology.

Our computations show that $\varphi$ can take arbitrarily large even values. Thus the behavior of $\varphi$ is qualitatively different from what was seen before in [1].

Theorem 1.1. Let $n \in\{2,4,8\}, e \geq c \geq 0$, with $c \neq 1$, and $\Sigma^{2 n}$ be a homotopy $2 n$-sphere. If $n=2$ assume further that $\Sigma^{4} \backslash \operatorname{int}\left(D^{4}\right)$ embeds smoothly into $S^{4}$, where $D^{4}$ is a smooth 4-disk. Then

$$
\varphi\left(\Sigma^{2 n} \#_{e} S^{n} \times S^{n} \#_{c} S^{1} \times S^{2 n-1}, \#_{c} S^{1} \times S^{n}\right)=2 e-2 c+2 .
$$

Here $\#_{c} S^{1} \times S^{n}=S^{n+1}$ if $c=0$ and $\#_{e} S^{n} \times S^{n} \#_{c} S^{1} \times S^{2 n-1}=S^{2 n}$ if $e=c=0$.

The structure of the proof of the theorem is as follows. We prove Proposition 2.1. which yields a lower bound for the number of critical values derived from topological obstructions of an algebraic nature. The existence of a non-trivial lower bound is not obvious since one might think that several singularities could combine into a single more complicated singularity. However, the proof uses only standard techniques of algebraic topology. The next step taken in section 3 is to construct explicit smooth maps with any even number of singularities. This follows by taking fiber sums of elementary blocks of maps coming naturally from Hopf fibrations. This construction is an immediate generalization of the one considered by Antonelli in the case of two elementary blocks in ([3, pp. 185-186). Then Proposition 3.1 concludes the proof. We warn the reader that our proof is narrative and key facts are singled out as lemmas in the process of unfolding the proof of these two propositions.

Remark 1.1. Observe that $S^{1} \times S^{2 n-1}$ fibers over $S^{1} \times S^{n}$ when $n \in\{2,4,8\}$ so that the formula from Theorem 1.1] is still valid for $\Sigma^{2 n}=S^{2 n}, e=0$ and $c=1$. However, we do not know how to evaluate $\varphi$ when $e \leq c-1$. The present methods do not work for $e \geq c=1$ either.

\section{A LOWER BOUND FOR THE NUMBER OF CRITICAL VALUES}

Proposition 2.1. For any dimension $n \geq 2$, homotopy $2 n$-sphere $\Sigma^{2 n}$ and nonnegative integers $e$ and $c$, with $c \neq 1$, we have:

$$
\varphi\left(\Sigma^{2 n} \#_{e} S^{n} \times S^{n} \#_{c} S^{1} \times S^{2 n-1}, \#_{c} S^{1} \times S^{n}\right) \geq 2 e-2 c+2 .
$$

Here $\#_{c} S^{1} \times S^{n}=S^{n+1}$ if $c=0$ and $\#_{e} S^{n} \times S^{n} \#_{c} S^{1} \times S^{2 n-1}=S^{2 n}$ if $e=c=0$.

We will prove, more generally, the following:

Proposition 2.2. Let $M^{2 n}$ and $N^{n+1}$ be closed connected orientable manifolds and $n \geq 2$. Assume that $\pi_{1}(M) \cong \pi_{1}(N)$ is a free group $\mathbb{F}(c)$ on $c$ generators, $c \neq 1$ (with $\mathbb{F}(0)=0), \pi_{j}(M)=\pi_{j}(N)=0$, for $2 \leq j \leq n-1$ and $H_{n-1}(M)=0$. Then $\varphi(M, N) \geq \beta_{n}(M)-2 c+2$, where $\beta_{k}$ denotes the $k$-th Betti number. 
Proof. Let $B=B(f)$ denote the set of critical values of a smooth map $f: M \rightarrow N$. We will prove that the cardinality $|B|$ of $B(f)$ satisfies $|B| \geq \beta_{n}(M)-2 c+2$, which will imply our claim. Set $V=f^{-1}(B(f)) \subset M$. We can assume that $f$ has finitely many critical points, since otherwise the claim of Proposition 2.2 would be obviously verified.

The following two lemmas do not depend on the homotopy assumptions of Proposition 2.2

Lemma 2.1. If $A$ is a non-empty finite subset of a connected closed orientable manifold $N^{n+1}$, then $\beta_{n}(N \backslash A)=\beta_{n}(N)+|A|-1$.

Proof. This is clear from the homology exact sequence of the pair $(N, N \backslash A)$.

Lemma 2.2. If $M^{n+q+1}$ and $N^{n+1}$ are smooth manifolds and $f: M \rightarrow N$ is a smooth map with finitely many critical points, then the inclusions $M \backslash V \hookrightarrow M$ and $N \backslash B \hookrightarrow N$ are $n$-connected.

Proof. This is obvious for $N \backslash B \hookrightarrow N$. It remains to prove that $\pi_{k}(M, M \backslash V) \cong 0$ for $k \leq n$. Take $\alpha:\left(D^{k}, S^{k-1}\right) \rightarrow(M, M \backslash V)$ to be an arbitrary smooth map of pairs. Since the critical set $C(f)$ of $f$ is finite and contained in $V$, there exists a small homotopy of $\alpha$ relative to the boundary such that the image $\alpha\left(D^{k}\right)$ avoids $C(f)$. By compactness there exists a neighborhood $U$ of $C(f)$ consisting of disjoint balls centered at the critical points such that $\alpha\left(D^{k}\right) \subset M \backslash U$. We can arrange by a small isotopy that $V$ becomes transversal to $\partial U$.

Observe further that $V \backslash U$ consists of regular points of $f$ and thus it is a properly embedded sub-manifold of $M \backslash U$. General transversality arguments show that $\alpha$ can be made transverse to $V \backslash U$ by a small homotopy. By dimension counting this means that $\alpha\left(D^{k}\right) \subset M \backslash U$ is disjoint from $V$ and thus the class of $\alpha$ in $\pi_{k}(M, M \backslash V)$ vanishes.

The restriction of $f$ to $M \backslash V$ is a proper submersion, and thus the restriction $\left.f\right|_{M \backslash V}$ is an open map. In particular, $f(M \backslash V) \subset N \backslash B$ is an open subset. On the other hand, the closed map lemma states that a proper map between locally compact Hausdorff spaces is also closed. Thus $f(M \backslash V)$ is also closed in $N \backslash B$ and hence $f(M \backslash V)=N \backslash B$. According to Ehresmann's theorem, the restriction $\left.f\right|_{M \backslash V}$ is then a locally trivial smooth fibration over $N \backslash B$ with compact smooth fiber $F^{n-1}$ (see [5]).

Lemma 2.3. Assume that $c \neq 1$. Then the generic fiber $F$ is homotopy equivalent to the $(n-1)$-sphere.

Proof. When $c=0$ the claim is a simple consequence of the homotopy sequence of the fibration $M \backslash V \rightarrow N \backslash B$.

Let us assume henceforth that $c \geq 2$. Consider the last terms of the homotopy exact sequence of this fibration:

$$
\rightarrow \pi_{1}(M \backslash V) \stackrel{f_{*}}{\rightarrow} \pi_{1}(N \backslash B) \stackrel{p}{\rightarrow} \pi_{0}(F) \rightarrow \pi_{0}(M \backslash V) \rightarrow \pi_{0}(N \backslash B) .
$$

From Lemma $2.2 M \backslash V$ and $N \backslash B$ are connected and $\pi_{1}(M \backslash V) \cong \pi_{1}(N \backslash B) \cong \mathbb{F}(c)$. If $F$ has $d \geq 2$ connected components, then the kernel ker $p$ of $p$ is a finite index proper subgroup of the free non-abelian group $\mathbb{F}(c)$. The Nielsen-Schreier theorem states that a subgroup of a free group is free. Moreover, the rank of an index $d$ subgroup of $\mathbb{F}(c)$ is $d(c-1)+1$, by the Schreier index formula. In particular ker $p$ 
is a free group of rank $d(c-1)+1$, where $d$ is the number of components of $F$, and hence its rank is larger than $c$. On the other hand, by exactness of the sequence above, $\operatorname{ker} p$ is also the image of $f_{*}$ and thus it is a group of rank at most $c$. This contradiction shows that $F$ is connected.

If $n=2$, then $F$ is a circle, as claimed.

Now let $n>2$. We obtained above that $f_{*}$ is surjective. Since finitely generated free groups are Hopfian any surjective homomorphism $\mathbb{F}(c) \rightarrow \mathbb{F}(c)$ is also injective. Since $\pi_{2}(N \backslash B) \cong \pi_{2}(N)=0$ and $f_{*}$ is injective we derive that $\pi_{1}(F)=0$. The remaining terms of the homotopy exact sequence of the fibration and Lemma 2.2 show then that $\pi_{j}(F)=0$ for $2 \leq j \leq n-2$. Thus $F$ is a homotopy sphere.

Lemma 2.4. Suppose that $B \neq \emptyset$.

(1) We have $H_{1}(N \backslash B) \cong \mathbb{Z}^{c}, H_{n}(N \backslash B)=\mathbb{Z}^{|B|+c-1}$ and $H_{n+1}(N \backslash B)=0$.

(2) If $n>2$, then $H_{n-1}(M \backslash V)=0$.

(3) The homomorphism $H_{n}(M \backslash V) \rightarrow H_{n}(M)$ induced by the inclusion map is surjective.

Proof. The first two assertions are consequences of Lemma 2.1. Lemma 2.2 and standard algebraic topology. For instance, $H_{1}(N \backslash B) \cong H_{1}(N)=\mathbb{Z}^{c}$. The last claim follows from Lemma 2.2 and the long exact sequence in homology of the pair $(M, M \backslash V)$.

Lemma 2.5. If $B \neq \emptyset$ and $c \neq 1$, then the rank of $H_{n}(M \backslash V)$ is $2 c+|B|-2$.

Proof. The Gysin sequence of the fibration $M \backslash V \rightarrow N \backslash B$ (whose fiber is a homotopy sphere) reads:

$$
\rightarrow H_{m}(M \backslash V) \rightarrow H_{m}(N \backslash B) \rightarrow H_{m-n}(N \backslash B) \rightarrow H_{m-1}(M \backslash V) \rightarrow
$$

Consider the exact subsequence

$$
\begin{aligned}
H_{n+1}(N \backslash B) \rightarrow H_{1}(N \backslash B) & \rightarrow H_{n}(M \backslash V) \rightarrow H_{n}(N \backslash B) \rightarrow H_{0}(N \backslash B) \\
& \rightarrow H_{n-1}(M \backslash V) .
\end{aligned}
$$

If $n>2$, then the first and the last terms vanish.

The Euler characteristic of this subsequence is zero by exactness, and thus the rank of $H_{n}(M \backslash V)$ is $2 c+|B|-2$ by Lemma 2.4.

When $n=2$, we can complete the exact sequence above by adding one more term to its right, namely $H_{1}(M \backslash V) \stackrel{f_{*}}{\rightarrow} H_{1}(N \backslash B)$. However, $f_{*}$ is actually the map induced in homology by the isomorphism $f_{*}: \pi_{1}(M) \rightarrow \pi_{1}(N)$ and is thus an isomorphism itself. The argument with the Euler characteristic can be applied again and yields the claimed result.

From Lemma 2.5 and Lemma 2.4 (3) we derive that

$$
2 c+|B|-2 \geq \beta_{n}(M)
$$

and the proposition is proved.

Corollary 2.1. If $M^{2 n}$ is a smooth $(n-1)$-connected closed manifold, then

$$
\varphi\left(M, \Sigma^{n+1}\right) \geq \beta_{n}(M)+2,
$$

where $\Sigma^{n+1}$ is a homotopy sphere. 
Remark 2.1. The present approach does not work for $c=1$. In fact, fibers might have several connected components, each one being a homotopy sphere. In the absence of an upper bound for the number of components, the Leray-Serre spectral sequence leads only to a trivial lower bound for the number of critical values.

\section{Fiber sums of SUSpensions of Hopf fibrations}

Proposition 3.1. Let $n \in\{2,4,8\}, e \geq c \geq 0$, with $c \neq 1$, and $\Sigma^{2 n}$ be a homotopy $2 n$-sphere. If $n=2$ assume further that $\Sigma^{4} \backslash \operatorname{int}\left(D^{4}\right)$ embeds smoothly into $S^{4}$, where $D^{4}$ is a smooth 4-disk. Then

$$
\varphi\left(\Sigma^{2 n} \#_{e} S^{n} \times S^{n} \#_{c} S^{1} \times S^{2 n-1}, \#_{c} S^{1} \times S^{n}\right) \leq 2 e-2 c+2 .
$$

Proof. Recall from [1] that $\varphi\left(S^{2 n}, S^{n+1}\right)=2$ if $n=2,4$ or 8 . This is realized by taking suspensions of both spaces in the Hopf fibration $h: S^{2 n-1} \rightarrow S^{n}$, where $n=2,4$ or 8 , and then smoothing the new map at both ends. The extension $H: S^{2 n} \rightarrow S^{n+1}$ has precisely two critical points. This is also the basic example of a Montgomery-Samelson fibration with finitely many singularities, as considered in [4. Antonelli has considered in [3] manifolds which admit maps with two critical points into spheres, by gluing together two copies of $H$.

Our aim is to define fiber sums of Hopf fibrations leading to other examples of pairs of manifolds with finite $\varphi$ using Antonelli's construction for more general gluing patterns. Identify $S^{n+1}$ (and respectively $S^{2 n}$ ) with the suspension of $S^{n}$ (respectively $S^{2 n-1}$ ) and thus equip it with the coordinates $(x, t)$, where $|x|^{2}+t^{2}=$ 1 , and $t \in[-1,1]$. We call the coordinate $t$ the height of the respective point. The suspension $H$ is then given by

$$
H(x, t)=\left(\psi(|x|) h\left(\frac{x}{|x|}\right), t\right),
$$

where $\psi:[0,1] \rightarrow[0,1]$ is a smooth increasing function infinitely flat at 0 such that $\psi(0)=0$ and $\psi(1)=1$.

Pick up a number of points $x_{1}, x_{2}, \ldots, x_{k} \in S^{n+1}$ and their small enough disk neighborhoods $x_{i} \in D_{i} \subset S^{n+1}$, such that

(1) the projections of $D_{i}$ on the height coordinate axis are disjoint;

(2) the $D_{i}$ 's do not contain the two poles; i.e. their projections on the height axis are contained in the open interval $(-1,1)$.

Let $A_{k}$ be the manifold with boundary obtained by deleting from $S^{n+1}$ of the interiors of the disks $D_{i}$, for $1 \leq i \leq k$. Also let $B_{k} \subset S^{2 n}$ denote the preimage of $A_{k}$ by the suspended Hopf map $H$. Since $H$ restricts to a trivial fibration over the disks $D_{i}$ it follows that $B_{k}$ is a manifold, each one of its boundary components being diffeomorphic to $S^{n-1} \times S^{n}$. Moreover, the boundary components are endowed with a natural trivialization induced from $D_{i}$.

Now let $\Gamma$ be a finite connected graph. To each vertex $v$ of valence $k$ we associate a block $\left(B_{v}, A_{v},\left.H\right|_{B_{v}}\right)$, which will be denoted $\left(B_{k}, A_{k},\left.H\right|_{B_{k}}\right)$, when we want to emphasize the dependence on the number of boundary components. Each boundary component of $A_{v}$ or $B_{v}$ corresponds to an edge incident to the vertex $v$. We define the fiber sum along $\Gamma$ as the following triple $\left(B_{\Gamma}, A_{\Gamma}, H_{\Gamma}\right)$ :

(1) $A_{\Gamma}$ is the result of gluing the manifolds with boundary $A_{v}$, associated to the vertices $v$ of $\Gamma$, by identifying for each edge $e$ joining the vertices $v$ and $w$ (which might coincide) the pair of boundary components in $A_{v}$ and 
$A_{w}$ corresponding to the edge $e$. The identification is made by using an orientation-reversing diffeomorphism of the boundary spheres.

(2) $B_{\Gamma}$ is the result of gluing the manifolds with boundary $B_{v}$, associated to the vertices $v$ of $\Gamma$, by identifying for each edge $e$ joining the vertices $v$ and $w$ (which might coincide) the boundary components in $B_{v}$ and $B_{w}$ corresponding to the pair of boundary components in $A_{\Gamma}$ associated to $e$. Gluings in $B_{\Gamma}$ are realized by some orientation-reversing diffeomorphisms which respect the product structure over boundaries of $A_{v}$ and $A_{w}$. We choose the identification diffeomorphism $\nu: \partial B_{v} \rightarrow \partial B_{w}$ to be the one from the construction of the double of $B_{v}$.

(3) As the boundary components are identified, the natural trivializations of the boundary components of $B_{v}$ agree in pairs. Thus the maps $H_{v}$ induce a well-defined map $H_{\Gamma}: B_{\Gamma} \rightarrow A_{\Gamma}$.

In the case where the graph $\Gamma$ consists of two vertices joined by an edge, this construction is essentially that given in ([3], pp. 185-186).

Proposition 3.2. The map $H_{\Gamma}: B_{\Gamma} \rightarrow A_{\Gamma}$ has $2 m$ critical points, where $m$ is the number of vertices of $\Gamma$.

Proof. This is clear by construction.

We say that $\Gamma$ has $c$ independent cycles if the rank of $H_{1}(\Gamma)$ is $c$. This is equivalent to asking $\Gamma$ to become a tree only after removal of at least $c$ edges. Moreover, $c=e-m+1$, where $e$ denotes the number of edges.

Proposition 3.3. If $\Gamma$ has e edges and c cycles, i.e. $e-c+1$ vertices, then $B_{\Gamma}$ is diffeomorphic to $\Sigma^{2 n} \#_{e} S^{n} \times S^{n} \#_{c} S^{1} \times S^{2 n-1}$ (where $\Sigma^{2 n}$ is a homotopy sphere, which is trivial when $n=2)$, while $A_{\Gamma}$ is diffeomorphic to $\#_{c} S^{1} \times S^{n}$. Here $\#{ }_{c} S^{1} \times S^{n}$ stands for $S^{n+1}$ when $c=0$.

Proof. The sub-blocks $A_{k}$ are diffeomorphic to the connected sum of $k$ copies of disks $D^{n+1}$ out of their boundaries. When gluing together two such distinct subblocks (since there is an edge in $\Gamma$ joining the corresponding vertices) the respective pair of disks leads to a factor $D^{n+1} \cup_{\mu} D^{n+1}$, where $\mu: S^{n} \rightarrow S^{n}$ is the identification map. If $\mu$ is a reflection, then the factor $D^{n+1} \cup_{\mu} D^{n+1}$ is the double of $D^{n+1}$ and hence diffeomorphic to $S^{n+1}$.

When gluing all sub-blocks in the pattern of the graph $\Gamma$, the only non-trivial contribution comes from the cycles. Each cycle of $\Gamma$ introduces a 1-handle. Thus the manifold $A_{\Gamma}$ is diffeomorphic to $\#_{c} S^{1} \times S^{n}$.

Further we have a similar result for the sub-blocks $B_{k}$ :

Lemma 3.1. The sub-blocks $B_{k}$ are diffeomorphic to the connected sum of $k$ copies of the product $S^{n} \times D^{n}$ out of their boundaries.

Proof. One obtains $B_{k}$ by deleting out $k$ copies of $H^{-1}\left(D_{i}\right)$; each $H^{-1}\left(D_{i}\right)$ is a tubular neighborhood of the (generic) fiber of $H$ and thus diffeomorphic to $S^{n-1} \times$ $D^{n+1}$.

When $k=1$ the generic fiber of $H$ is an $S^{n-1}$ embedded in $S^{2 n}$, namely, the image of the fiber of the Hopf fibration in the suspension sphere $S^{2 n}$. The generic fiber is unknotted in $S^{2 n}$, as an immediate consequence of Haefliger's classification of smooth embeddings. In fact, according to [7, any smooth embedding of $S^{k}$ in $S^{m}$ is unknotted, i.e. isotopic to the boundary of a standard ball, if the dimensions 
satisfy the meta-stable range condition $k<\frac{2}{3} m-1$. This implies that the complement of a regular neighborhood of the fiber is diffeomorphic to the complement of a standard sphere and thus to $S^{n} \times D^{n}$.

When $k \geq 2$ we remark that the fibers over the points $x_{i} \in D_{i}$ lie at different heights and thus they are contained in disjoint slice spheres of the suspension $S^{2 n}$. This implies that these fibers are unlinked, i.e. isotopic to the boundary of a set of disjoint standard balls. Thus the complement of a regular neighborhood of their union is diffeomorphic to the connected sum of their individual complements and therefore to the connected sum of $k$ copies of the product $S^{n} \times D^{n}$ out of their boundaries.

Let us stick for the moment to the case when $k=1$ and we have two diffeomorphic sub-blocks $B_{v}$ and $B_{w}$, each one having one boundary component, to be glued together. Recall that the identification diffeomorphism $\nu: \partial B_{v} \rightarrow \partial B_{w}$ is the one from the construction of the double of $B_{v}$. Observe that the maps $B_{v} \rightarrow A_{v}$ and $B_{w} \rightarrow A_{w}$ glue together to form a well-defined smooth map $B_{v} \cup_{\nu} B_{w} \rightarrow A_{v} \cup_{\mu} A_{w}$, as already noticed in ([3], p. 185).

Lemma 3.2. The factor $B_{v} \cup_{\nu} B_{w}$ is diffeomorphic to $\Sigma^{2 n} \# S^{n} \times S^{n}$, where $\Sigma^{4}=$ $S^{4}$.

Proof. Consider first the case $n=2$, which is the most interesting one since the result cannot follow from general classification results. The sub-block $D^{2} \times S^{2}$ can be easily described by a Kirby diagram (see [6], chapter 4), which encodes its handlebody structure. As $D^{2} \times S^{2}$ is obtained from $D^{4}$ by throwing away the regular neighborhood of an unknotted circle (i.e. a 1-handle) it can be described as the result of attaching the dual 2-handle on an unknotted circle with framing 0 . There is also a dual handlebody decomposition of $D^{2} \times S^{2}$ in which each $j$-handle generates a $(4-j)$-handle. The double of $D^{2} \times S^{2}$ is then described by putting together the two handlebody descriptions (the usual one and the dual one) and thus is made of $D^{4}$ with two 2-handles and finally a 4-handle capping off the boundary component.

Attaching maps of 4-handles are orientation-preserving diffeomorphisms of $S^{3}$, and by a classical result of Cerf these are isotopic to the identity. Thus there exists a unique way to attach a 4 -handle to a 4 -manifold with boundary $S^{3}$. By the way, recall that a theorem of Laudenbach and Poenaru ([1]) shows that there is only one way up to global diffeomorphism to attach 3-handles and 4-handles to a 4-manifold with boundary $\#_{k} S^{1} \times S^{2}$ in order to obtain a closed manifold.

Now it is easy to see that the new 2-handle (in the handlebody structure of the double of $D^{2} \times S^{2}$ ) is attached along a meridian circle of the former 2-handle with 0 framing. Thus a Kirby diagram of the double of $D^{2} \times S^{2}$ consists of a Hopf link with both components having framing 0 , and it is well-known that this diagram is also that of $S^{2} \times S^{2}$. See also ([6], Example 4.6.3) for more details.

This argument applies as well for $n \geq 3$. We have a handle decomposition of $D^{n} \times S^{n}$ as $D^{2 n}$ with one $n$-handle attached. The set of framings on a sphere $S^{n-1}$ in $\partial D^{2 n}$ is acted upon freely transitively by $\pi_{n-1}(O(n))$. Moreover $\pi_{3}(O(4)) \cong$ $\pi_{7}(O(8)) \cong \mathbb{Z} \oplus \mathbb{Z}$ (see [12]). Then the $n$-handle is attached on an unknotted $(n-1)$-sphere with trivial framing, i.e. the $(0,0)$-framing. Observe that this is the canonical framing associated to the identity attaching map $\mathrm{id}_{S^{n-1} \times D^{n}}$ (see e.g. 6], Example 4.1.4.(d)). Further the double of $D^{n} \times S^{n}$ is obtained by putting together 
the usual handlebody and its dual. As above we can describe the double as the result of attaching two $n$-handles and one $2 n$-handle. The dual $n$-handle is attached on a meridian $(n-1)$-sphere which links once the former attaching $(n-1)$-sphere and has trivial framing. The union of the two spheres is the analogue of the Hopf link in $S^{2 n-1}=\partial D^{2 n}$. As is well known $S^{n} \times S^{n}$ can also be obtained by adding two $n$-handles along this high-dimensional trivially-framed Hopf link and a $2 n$-handle.

The only difference between the cases $n>2$ and $n=2$ is that the result of attaching a $2 n$-handle for $n>2$ is not unique, as there might exist diffeomorphisms of $S^{2 n-1}$ which are not isotopic to the identity. However, detaching and then reattaching a $2 n$-handle with a reflection diffeomorphism as gluing map will create an exotic sphere (for $n \geq 4$ ), and thus the double is diffeomorphic to $\Sigma^{2 n} \# S^{n} \times S^{n}$ for some homotopy sphere $\Sigma^{2 n}$.

When gluing all sub-blocks in the pattern of the graph $\Gamma$ such that each identification map is $\nu$, then each pair of sub-blocks determines a factor $\Sigma^{2 n} \# S^{n} \times S^{n}$. If there are no cycles in $\Gamma$, then we obtain a connected sum of such factors, namely $\Sigma^{2 n} \#_{e} S^{n} \times S^{n}$. Finally, the only additional non-trivial contribution comes from the cycles. Each cycle of $\Gamma$ introduces an extra 1-handle. Thus the manifold $B_{\Gamma}$ is diffeomorphic to $\Sigma^{2 n} \#_{e} S^{n} \times S^{n} \#_{c} S^{1} \times S^{2 n-1}$.

In order to prove Proposition 3.1 it suffices now to show that one can attach a homotopy sphere $\Sigma$ to the manifolds $B_{\Gamma}$ and still have the same number of critical points.

Every homotopy $m$-sphere $\Sigma^{m}$, for $m \neq 4$, can be obtained as the union of two disks glued together along their boundaries using some diffeomorphism $f$ of the $(m-1)$-sphere. Therefore, by removing a small disk centered at a critical point and then gluing it back using the diffeomorphism $f$, the manifold changes by means of a connected sum with the homotopy sphere $\Sigma^{m}$. When $m=4$ it is unknown whether all homotopy 4-spheres can be obtained as the union of two disks. Actually, if this were true, then any homotopy 4-sphere would be diffeomorphic to the standard 4-sphere. But we can obtain any homotopy 4-sphere as the gluing of one disk and a homotopy 4 -disk. Consider a homotopy 4 -sphere $\Sigma^{4}$ for which the associated homotopy 4 -disk $\Delta^{4}$ embeds smoothly into $S^{4}$. Then, as above, by removing a small disk centered at a critical point and then gluing the homotopy 4disk $\Delta^{4}$ along the boundary 3 -sphere, we see that the 4-manifold changes by means of a connected sum with the homotopy sphere $\Sigma^{4}$.

Since the homotopy spheres form a finite abelian group under the connected sum, one can obtain in this way all manifolds of the form $\Sigma^{2 n} \#_{e} S^{n} \times S^{n} \#_{c} S^{1} \times S^{2 n-1}$, when $n \neq 2$, and respectively those for which $\Sigma^{4} \backslash D^{4}$ embeds smoothly into $S^{4}$, when $n=2$.

One shows (see 1], where this argument is carried out in detail) that we can glue together the two restrictions of the smooth map to the disk and respectively to its complementary disk in order to obtain a smooth map on the connected sum $B_{\Gamma} \# \Sigma$ with the same (non-zero) number of critical points, namely, $2 e-2 c+2$. When $n=2$ we need a theorem of Huebsch and Morse for $n=2$ (see [8]) concerning the existence of smooth maps $\Delta^{4} \rightarrow D^{4}$ with one critical point, for a homotopy 4-disk $\Delta^{4}$ which embeds smoothly into $S^{4}$. 
Remark 3.1. Recall that the group $\Theta^{k}$ of homotopy $k$-spheres is $\Theta^{k}=\mathbb{Z} / 2 \mathbb{Z}$ when $k \in\{8,16\}$.

Remark 3.2. By twisting $\mu$ by a diffeomorphism of $S^{n}$ which is not isotopic to the identity (e.g. when $n=8$ ), one could obtain exotic sphere factors in $A_{\Gamma}$. More interesting examples correspond to twisting $\nu$ by some orientation-preserving diffeomorphism $\eta: S^{n-1} \times S^{n} \rightarrow S^{n-1} \times S^{n}$ which still respect the product structure. For instance we can consider some $\eta$ induced from a map $S^{n-1} \rightarrow S O(n+1)$ whose homotopy class is an element of $\pi_{n-1}(S O(n+1))$. It seems that all examples obtained by twisting are still diffeomorphic to $\Sigma^{2 n} \#_{e} S^{n} \times S^{n} \#_{c} S^{1} \times S^{2 n-1}$.

\section{EXAmples With $\varphi=1$}

The result of [1] shows that if $\varphi\left(M^{m}, N^{n+1}\right)$ is finite non-zero (small codimension, non-exceptional dimensions), then $\varphi\left(M^{m}, N^{n+1}\right)=1$ and $M^{m}$ should be diffeomorphic to $\Sigma^{m} \# \widehat{N}$, where $\Sigma^{m}$ is an exotic sphere and $\widehat{N}$ is the total space of a smooth fibration, such that $M^{m}$ is not fibered over $N$. Actually this construction might produce non-trivial examples in any codimension.

Proposition 4.1. If $\Sigma^{m}$ is an exotic sphere (for $m=4$ we assume that $\Sigma^{4} \backslash \operatorname{int}\left(D^{4}\right)$ embeds smoothly in $\left.S^{4}\right)$ and if $\widehat{N} \rightarrow N$ is a smooth fibration, then $\varphi\left(\Sigma^{m} \# \widehat{N}, N\right) \in$ $\{0,1\}$.

Proof. We obtain $\Sigma^{m} \# \widehat{N}$ from $\widehat{N}$ by excising a ball $D^{n+1}$ and gluing it (or a homotopy 4-disk when $m=4$ ) back by means of a suitable diffeomorphism $h$ of its boundary. By a classical result of Huebsch and Morse (8), there exists a smooth homeomorphism $\Sigma^{m} \# \widehat{N} \rightarrow \widehat{N}$ which has only one critical point located in the ball $D^{n+1}$. This provides a smooth map $\Sigma^{m} \# \widehat{N} \rightarrow N$ with one critical point.

Remark 4.1. Notice however that $\Sigma^{m} \# \widehat{N}$ might still be fibered over $N$, although not diffeomorphic to $\widehat{N}$. This is so when $\widehat{N} \rightarrow N$ is the Hopf fibration $S^{7} \rightarrow S^{4}$ and $\Sigma^{7} \# \widehat{N}$ is a Milnor exotic sphere, namely, an $S^{3}$-fibration over $S^{4}$ with Euler class \pm 1 .

Remark 4.2. The manifold $M^{m}=\Sigma^{m} \# S^{m-n-1} \times S^{n+1}$ is not diffeomorphic to $S^{m-n-1} \times S^{n+1}$ if $\Sigma^{m}$ is an exotic sphere (see [13). Thus, the proposition above yields effective examples where $\varphi=1$.

If $\Sigma^{8}$ is the exotic 8-sphere which generates the group $\Theta^{8}=\mathbb{Z} / 2 \mathbb{Z}$, then $\varphi\left(\Sigma^{8} \# S^{3}\right.$ $\left.\times S^{5}, S^{5}\right)=1$. In fact $M^{8}=\Sigma^{8} \# S^{3} \times S^{5}$ is homeomorphic but not diffeomorphic to $S^{3} \times S^{5}$. Assume the contrary, namely, that $M^{8}$ smoothly fibers over $S^{5}$. Then the fiber should be a homotopy 3 -sphere and hence $S^{3}$ by the Poincare Conjecture. The $S^{3}$-fibrations over $S^{5}$ are classified by the elements of $\pi_{4}(S O(4)) \cong \mathbb{Z} / 2 \mathbb{Z} \oplus \mathbb{Z} / 2 \mathbb{Z}$. There exist precisely two homotopy types among the $S^{3}$-fibrations over $S^{5}$ which admit cross-sections (see [9] p. 217). If $M^{8}$ is a $S^{3}$-fibration, then it should have a cross-section, because it is homotopy equivalent to $S^{3} \times S^{5}$ and the existence of a cross-section is a homotopy invariant (see [9, p. 196, 10], p. 164). However the two homotopy types correspond to two distinct isomorphism types as spheres bundles. In fact they are classified by the image of $\pi_{4}(S O(3)) \cong \mathbb{Z} / 2 \mathbb{Z}$ into $\pi_{4}(S O(4))$. 
This means that an $S^{3}$-fibration having a cross-section is either homotopy equivalent to the trivial fibration and then it is isomorphic to the trivial fibration or else it does not have the same homotopy type as $S^{3} \times S^{5}$. Observe also that there is only one $O(4)$-equivalence class and thus precisely two isomorphism classes of such $S^{3}$-fibrations without cross-sections $\left([10]\right.$, p. 164). In particular, non-trivial $S^{3}$ fibrations over $S^{5}$ cannot be homeomorphic to $M^{8}$, and this contradiction shows that $M^{8}$ cannot smoothly fiber over $S^{5}$.

\section{ACKNOWLEDGEMENTS}

The authors are indebted to Dennis Sullivan, Yuli Rudyak and András Szücs for useful discussions on this topic during the M. M. Postnikov Memorial Conference at Bedlewo, June 2007. The first author was partially supported by the ANR Repsurf: ANR-06-BLAN-0311. The second author was partially supported by the CNCSIS grant of type A, 8/1467/2007 and partially by the CNCSIS grant PN II, ID $523 / 2007$. The third author was partially supported by Eastern Mediterranean University Scientific Research Budget BAP-A-08-05. The third author thanks Jianzhong Pan, Haibao Duan and Kaiming Zhao of the Institute of Mathematics of CAS in Beijing for their hospitality when part of this paper was written in the summer of 2007.

\section{REFERENCES}

1. D. Andrica and L. Funar, On smooth maps with finitely many critical points, J. London Math. Soc. (2) 69(2004), 783-800; Addendum, ibid. 73(2006), 231-236. MR2050046 (2005f:57043) MR $2197380(2007 \mathrm{~b}: 57059)$

2. D. Andrica, L. Funar and E. Kudryavtseva, The minimal number of critical points of maps between closed manifolds, Russian Journal of Mathematical Physics, special issue for the conference celebrating the 60th birthday of Nicolae Teleman (Ancona and Porto Nuovo, September 2007), J.-P. Brasslet, A. Legrand, R. Longo, A. Mishchenko, editors, to appear.

3. P. L. Antonelli, Structure theory for Montgomery-Samelson fiberings between manifolds, I, II. Canad. J. Math. 21(1969), 170-179; ibid. 21(1969), 180-186. MR0238320 (38:6596)

4. P. L. Antonelli, Differentiable Montgomery-Samelson fiberings with finite singular sets, Canad. J. Math. 21(1969), 1489-1495. MR0261624 (41:6237)

5. A. Dimca, Singularities and topology of hypersurfaces, Springer-Verlag, Berlin-New York, 1992. MR.1194180 (94b:32058)

6. R. E. Gompf and A. I. Stipsicz, 4-manifolds and Kirby calculus, Graduate Studies in Mathematics, 20, American Mathematical Society, Providence, RI, 1999. MR:1707327(2000h:57038)

7. A. Haefliger, Differentiable embeddings of $S^{n}$ in $S^{n+q}$ for $q>2$, Ann. of Math. (2) 83(1966), 402-436. MR0202151 (34:2024)

8. W. Huebsch and M. Morse, Schoenflies extensions without interior differential singularities, Ann. of Math. (2) 76(1962), 18-54. MR0146847 (26:4366)

9. I. M. James and J. H. C. Whitehead, The homotopy theory of sphere bundles over spheres. I, Proc. London Math. Soc. (3) 4(1954), 196-218. MR0061838 (15:892b)

10. I. M. James and J. H. C. Whitehead, The homotopy theory of sphere bundles over spheres. II, Proc. London Math. Soc. (3) 5(1955), 148-166. MR0068836 (16:948d)

11. F. Laudenbach and V. Poénaru, A note on 4-dimensional handlebodies, Bull. Soc. Math. France 100(1972), 337-344. MR0317343 (47:5890)

12. M. Mimura, Homotopy theory of Lie groups, Handbook of algebraic topology (I. M. James, editor), 951-991, North-Holland, Amsterdam, 1995. MR.1361904(97c:57038) 
13. R. Schultz, On the inertia group of a product of spheres, Trans. Amer. Math. Soc. 156(1971), 137-153. MR0275453 (43:1209)

14. J. G. Timourian, Fiber bundles with discrete singular set, J. Math. Mech. 18(1968/69), 61-70. MR 0235571(38:3875)

Institut Fourier BP 74, UMR 5582, Université de Grenoble I, 38402 Saint-MartinD'HÈres CEDEX, France

E-mail address: funar@fourier.ujf-grenoble.fr

Department of Geometry, "Babeş-Bolyai" University, 400084 M. Kogălniceanu 1, Cluj-Napoca, Romania

E-mail address: cpintea@math.ubbcluj.ro

Department of Mathematics, Eastern Mediterranean University, Gazima GuUsa, North Cyprus, via Mersin 10, Turkey

E-mail address: ping.zhang@emu.edu.tr 